\title{
DEVELOPMENT OF A HYBRID DEVICE BASED ON INFRARED AND ULTRASONIC SENSORS FOR HUMAN RESOURCE MANAGEMENT
}

\author{
Joo-Heon Kim \\ Graduate Student, \\ Department of Civil, Environmental and \\ Architectural Engineering, \\ Korea University, Anam-dong, Seongbuk-gu, \\ Seoul, Korea \\ ringdoves@korea.ac.kr \\ Hun-Hee Cho \\ Assistant Professor, \\ Division of Architecture and Ocean Space, \\ Korea Maritime University, \\ Dongsam-dong, Youngdo-gu, \\ Busan, Korea \\ hhcho@hhu.ac.kr
}

\author{
Ung-Kyun Lee \\ $\mathrm{Ph}$. D. Candidate, \\ Department of Civil, Environmental and \\ Architectural Engineering, \\ Korea University, Anam-dong, Seongbuk-gu, \\ Seoul, Korea \\ uklee@korea.ac.kr \\ Kyung-In Kang \\ Professor, \\ Department of Civil, Environmental and \\ Architectural Engineering, \\ Korea University, Anam-dong, Seongbuk-gu, \\ Seoul, Korea \\ kikang@korea.ac.kr
}

\begin{abstract}
Human resources are continuously gaining importance in association with construction site management. With increasing interest being shown in the use of state-of-the-art technologies such as radio frequency identification (RFID) in site management, we developed a device based on infrared radiation and ultrasonic sensors for application in the management of human resources, especially labour safety management. We tested sensors and optimized the sensing range and angle of the device, which could detect workers using an infrared sensor that senses body heat and an ultrasonic sensor to measure the object's distance. The device allows a manager to determine where workers are, and how far they are from the point of sensing. This paper shows that the device can prevent fatal accidents such as falls. In doing so, it addresses a key issue in the management of safety on work sites.
\end{abstract}

\section{KEYWORDS}

Safety Management, Infrared Sensor, Ultrasonic Sensor

\section{INTRODUCTION}

Human resource management has been identified as very important for site management compared with such management at other locations. Because the management of the workforce has a direct impact on project performance, many researchers have presented management methods. For example, Brandenburg (2006) presented a management strategy for human resources to improve overall productivity, including labour safety and the success of the project [1]. Thomas (2006) suggested fundamental principles of workforce management for utilizing the workforce efficiently [2].

Hardware approaches to management methods are limited, especially in labour safety management, 
which occupies an important position in human resource management. To manage labour safety, many researchers have developed a coding system to facilitate the categorization of fatal falls to determine the importance of contributing factors and to derive effective protection strategies [3]. They have determined the causes of construction fall accidents and identified any particular patterns related to fall accidents [4]. However, there are limited techniques for finding out whether workers are exposed to danger and how far they are from a point of danger. Such a technique would allow labour safety to be managed directly and actively.

Therefore, we propose a state-of-the-art device combining infrared (IR) and ultrasonic (US) sensors for labour safety management on construction sites. This device is remarkably useful in detecting careless workers exposed to dangerous situations and making them aware of the danger, thereby reducing accidents resulting in fatalities. To identify the capacity and performance of the device, as shown in Figure 1, we tested it as follows. (1) We collected background information about IR and US sensors to discover their technical features and application status. (2) We proposed an approach to develop the device based on infrared radiation and ultrasonic technologies. (3) We optimized the sensing range and angle of the device that can detect workers, with the IR sensor sensing body heat and the US sensor measuring distance. (4) Finally, we present the feasibility of the detecting device combined with US and IR sensors (DEUI) to apply it to managing labour safety.

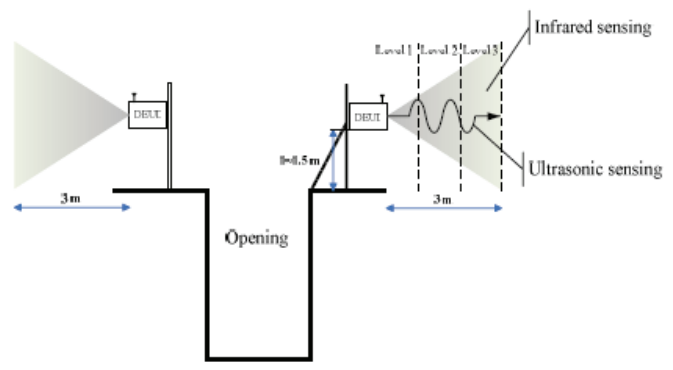

Figure 1 Performance of the Device

\section{BACKGROUND}

\subsection{Ultrasonic (US) Sensor}

US technology covers an enormous range of human endeavour, from non-destructive testing (NDT) to medical scanning; from chemical analysis to (alleged) nobbling of race horse; from welding to flowmeters [5].

US sensors utilize transducers that transform an electrical signal into a US wave and vice versa; in other words, the basic idea is that they transmit acoustic waves and receive waves after interaction between the US wave and the investigated process [6], [7]. Ultrasound covers a frequency range from $20 \mathrm{kHz}$ to about $1 \mathrm{GHz}$. For technical applications, the range $20 \mathrm{kHz}$ to 10 $\mathrm{MHz}$ is the most important.

Hauptmann (2002) classified US sensors and presented their features: non-invasive measurement, inline measurement, rapid response, low power consumption, excellent long-term stability, and high resolution and accuracy [7]. With the features, he also presented various applications of US sensors such as temperature measurement, pressure measurement, level and distance measurement, and flow measurement.

We used US sensors to determine the presence of workers and to measure their distance from the sensor. US has general application to level measurement, proximity detection, and robotics.

\subsection{Infrared (IR) Radiation}

IR sensors are widely used as proximity sensors and offer lower cost and faster response times than US sensors [8]. The coming of age of IR technology has opened the door to a variety of applications in electrooptic devices, premises security systems, communication systems, data transmission systems, missile warning systems, pollution monitoring sensors, and so on [9].

IR sensors can be classified as photon sensors or thermal sensors. In a photon sensor, the absorbed radiation excites electronic transitions within the material and the observed electrical output signal results in some way from the changed electronic energy distribution, while in a thermal sensor the incident radiation is absorbed and changes the temperature of the material, the signal being 
observed as a change in some temperaturedependent property of the material [10].

In this paper, a pyroelectric infrared ray (PIR) sensor was used, a type of thermal sensor that detects infrared radiation from body heat and is of general application to security or households.

\section{DEVICE CONFIGURATIONS}

The DEUI is an enhanced safety supervision method that can prevent fatal accidents such as falls. It consists of ultrasonic and infrared sensors, a speaker, and a main board. As shown in Figure 2, they can be operated by a rechargeable battery.

\subsection{Ultrasonic (US) Sensor}

US sensors have been used to determine the presence of workers. For testing, SensComp's 600 Series Instrument Transducer for operation in air at ultrasonic frequencies was used [12]. One reason why this sensor was chosen is that it is very applicable as it can detect soft targets such as clothes without absorption. It has a beam angle of $15^{\circ}$ and features ranging capability from $2.5 \mathrm{~cm}$ to $15.2 \mathrm{~m}$.

\subsection{Infrared (IR) Sensor}

It is important for US sensors to determine the presence of people when measuring distance, not other objects such as steels or other materials. Therefore, it is necessary that an IR sensor determines whether an object is human or not by sensing body heat.

In this test, Vessco's PIS-700 passive IR detector was used to sense IR radiation from body heat. It is of general application to security or households [13]. Its maximum coverage angle is $130^{\circ}$ and its sensitivity can be controlled.

\subsection{Recorder, Speaker and Main Board}

The device consists of sensors and a speaker, which can send a beep or a spoken message to people approaching the device, depending on the distance measured by US sensors from it. The voice or any other sound can be recorded very easily.

We divided the controlled space into three zones. The first zone is $2-3 \mathrm{~m}$ from the device, the second zone is $1-2 \mathrm{~m}$ from it, and the third zone is $0-1 \mathrm{~m}$ from it. When a worker is in the first zone, the speaker beeps. If the worker enters the second zone, it beeps faster. Finally, when the worker is in the third zone, the speaker sends a recorded voice message. This should alert a person approaching the DEUI to his or her increasingly dangerous situation.

The main board controls the interaction between the two sensors: when the IR sensor detects a person, the US sensors measures his or her distance from the DEUI, and the main board makes the speaker send out a recorded voice message such as "STOP".

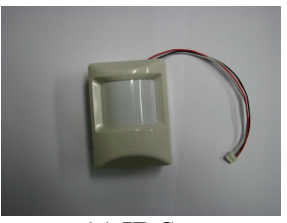

(a) IR Sensor

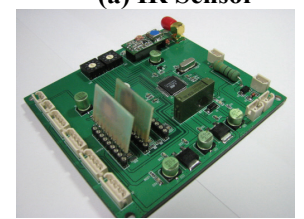

(c) Main Board

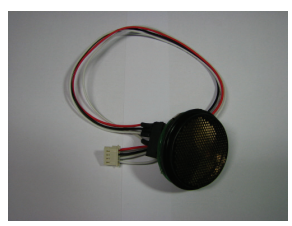

(b) US Sensor

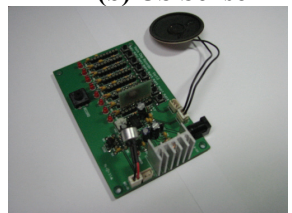

(d) Recorder and Speaker
Figure 2 Device Configurations

\section{TESTS}

\subsection{Test Plan}

The features of the two sensors such as sensing angle and range and their interactions in some situations are considered to optimize their incorporation in the device. We also tested the point at which the speaker should broadcast its voice message to find the detected distance from the DEUI, as shown in Figure 3. The following tests were conducted:

(1) Scenario test

(2) Optimizing range of sensors test

(3) Classifying detected zones test

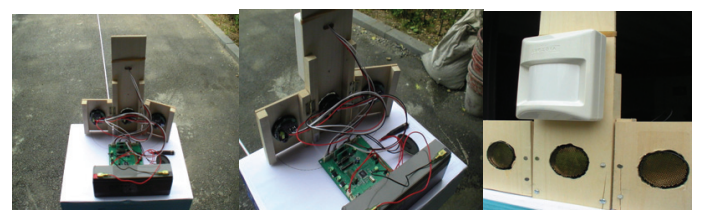

Figure 3 Test Model 


\subsection{Scenario Test}

The purpose of the scenario test is to investigate whether the US sensor detects obstacles such as walls rather than people. Therefore, some conditions were imposed as illustrated in Figure 4: near an opening with a wall on one side; near an opening with walls on two sides; or near an opening with no walls.

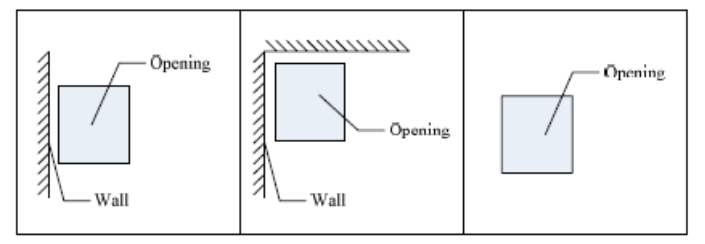

Figure 4 Test Conditions

\subsection{The Sensor Range Test}

The IR sensor was tested to confirm that it could sense body heat at ranges up to $3 \mathrm{~m}$. A man moved very slowly while we checked the point at which the sensor could detect him. When he was detected, a light was turned on. Using the light, the range and angle of the IR sensor were checked.

The US sensor was set up to detect the presence of a tester and to measure the distance. It was configured to detect a person after the IR had sensed body heat. When the sensor had detected a person, the speaker broadcast a recorded beep and a voice message. Thus, we could check the range and detection angle of the US sensor by using the sound. Considering the US sensor's optimal angle, three US sensors were used with one IR sensor.

\subsection{Classifying Detected Zones}

The purpose of this test is to see how quickly the DEUI could detect a person and the point at which the person was detected. The DEUI was set up so that when it detected a person, it sent out a different recorded sound or voice depending on the zone where the person was detected. Three detection zones were used: $0-1 \mathrm{~m}, 1-2 \mathrm{~m}$, and 2$3 \mathrm{~m}$ from the device. We checked that different sound and voice signals were sent out for detection in each zone, to indicate the danger level. We used this test to optimize the detection zones.

\section{RESULTS AND DISCUSSION}

We tested the DEUI to alert careless workers to dangerous situations. The test showed that the IR sensor had an angle of $120^{\circ}$ and detected a tester up to $3 \mathrm{~m}$ from the device. The optimal detecting distance was determined to be about $3 \mathrm{~m}$ away from the device because it should detect workers before they are very close to dangerous places.

The US sensors, on the other hand, had sensing angles of $15^{\circ}$ each, and as illustrated in Figure 5(b), we controlled their angles to be the same as the IR sensor's. When the three US sensors were adjusted to adapt to the IR sensor's angle, a dead zone was found within the range of the IR sensor. However, the device seldom recognized the dead zone when a tester was moving. Therefore, the US sensors' coverage was adjusted to about $120^{\circ}$, like the IR sensor's, as shown in Figure 5(a).

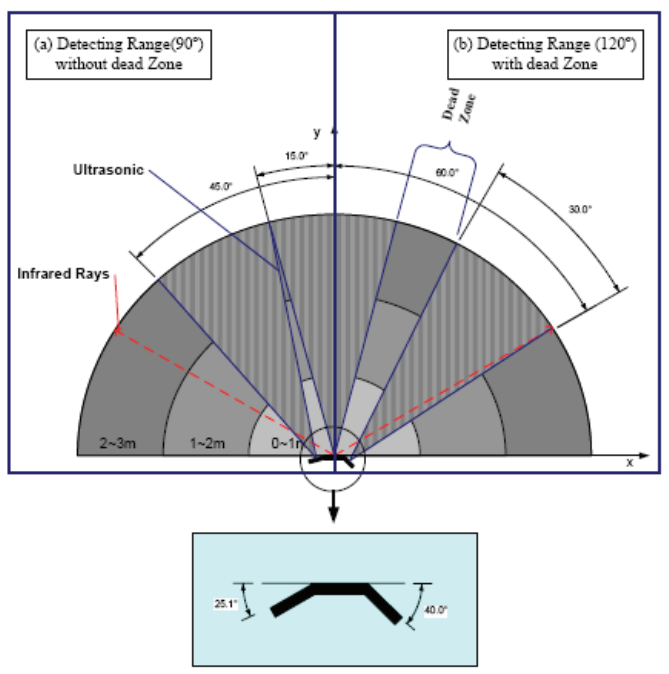

Figure 5 The Results of the Test

When the IR sensor detected a tester's body heat, the US sensors operated to detect his distance and the speaker warned him depending on the zone. However, the US sensors did not operate when the IR sensor could not detect body heat. That meant that although the DEUI was blocked by walls or other materials, the US sensors could not operate, and the speaker remained quiet because no body heat was sensed by the IR sensor. However there was limitation that when IR sensor sensed body heat, US sensor detected not only human but also other objects and their distance 
such as a wall and sent out the recorded sound regardless of human's location.

The results show that the DEUI can be applied to labour safety management. It detects workers who are approaching dangerous places such as water purifier tanks or openings in the construction site and alerts them to the dangerous situation. It can be expected that this active method will help reduce fall accidents and will help site managers supervise safety in the construction industry. Furthermore, it can be operated by a rechargeable battery and thus it can be set up anywhere very easily. Moreover, it should be possible to notify safety managers of detected dangerous situations via wireless data acquisition technologies such as radio frequency modems. This will make labour safety management effective and will help improve human resource management at construction sites.

\section{CONCLUSION}

This paper tested a DEUI to detect workers exposed to dangers on construction sites and to alert them to their situation. Our tests anticipated an active method to prevent accidents on construction sites, and our device could contribute to labour safety. Even though workers may not pay careful attention to dangerous situations, the device can prevent fatal accidents such as falls.

\section{ACKNOWLEDGMENT}

This work was supported by the Second Brain Korea 21 Project.

\section{REFERENCES}

[1] Brandenburg, S. G., Hass, C. T. \& Byrom, K. (2006) Strategic Management of Human Resources in Construction, Journal of Management in Engineering, ASCE, Vol. 22, No. 2, 89-96.

[2] Thomas, H. R. \& Horman, M. J. (2006) Fundamental Principles of Workforce Management, Journal of Construction Engineering and Management, Vol. 132, No. 1, 97-104.
[3] Chi C. F., Chang T. C. and Ting H. I. (2005) Accident patterns and prevention measures for fatal occupational falls in the construction industry, Applied Ergonomics, Vol. 36, Issue 4, 391-400,.

[4] Huang X. and Hinze J. (2003) Analysis of Construction Worker Fall Accidents, Journal of Construction Engineering and Management, Vol. 129, No. 3, 262-271.

[5] Asher R. C. (1997) Ultrasonic Sensors for Chemical and Process Plant, Institute of Physics Publishing, London.

[6] Hauptmann P., Lucklum R., Püttmer A. and Henning B. (1998) Ultrasonic sensors for process monitoring and chemical analysis: state-of-the-art and trends, Sensors and Actuators A: Physical, Volume 67, Issues 1-3, 32-48.

[7] Hauptmann P., Hoppe N. and Püttmer A. (2002) Application of ultrasonic sensors in the process industry, Measurement science \& technology, Vol. 13, No. 8, R73-R83

[8] Benet G., Blanes J. E., Simó J. E. and Pérez P. (2002) Using infrared sensors for distance measurement in mobile robots, Robotics and Autonomous Systems, Vol. 40, Issue 4, 255266

[9] Jha A. R. (2002) Infrared Technology Applications to Electrooptics, Photonic Devices, and Sensors, A Wiley-Interscience Publication, New York.

[10] Capper P. and Elliott C. T. (2001) Infrared Detectors and Emitters: Materials and Devices, Kluwer Academic Publishers, London.

[11] Nagarajan S., Banerjee P., Chen W. and Chin B. A. (1992) Control of the Welding Process Using Infrared Sensors, IEEE Transactions on Robotics and Automation, Vol. 8, No. 1, 8693

[12] www.senscomp.com

[13] www.vessco.co.kr 